\title{
Kernos
}

Revue internationale et pluridisciplinaire de religion grecque antique

$15 \mid 2002$

Varia

\section{Le culte des Grands Dieux de Samothrace à la période hellénistique}

\section{Zlatozara Gočeva}

\section{OpenEdition \\ Journals}

Édition électronique

URL : http://journals.openedition.org/kernos/1390

DOI : 10.4000/kernos.1390

ISSN : 2034-7871

Éditeur

Centre international d'étude de la religion grecque antique

Édition imprimée

Date de publication : 1 janvier 2002

ISSN : 0776-3824

Référence électronique

Zlatozara Gočeva, «Le culte des Grands Dieux de Samothrace à la période hellénistique », Kernos [En ligne], 15 | 2002, mis en ligne le 21 avril 2011, consulté le 30 avril 2019. URL : http:// journals.openedition.org/kernos/1390 ; DOI : 10.4000/kernos.1390 


\section{Le culte des Grands Dieux de Samothrace à la période hellénistique}

Le sanctuaire de Samothrace et les mystères qui y étaient célébrés soulèvent depuis longtemps beaucoup de problèmes ardus. C'est tout d'abord parce que les témoignages écrits sur ce sujet dans la littérature antique sont très vagues et controversés ${ }^{1}$. Ils sont, dans une large mesure, difficiles à interpréter à cause, avant tout, de l'interdiction de parler ouvertement de ces mystères et de leur caractère. D'autre part, des controverses existent quant à leur origine, leur datation et leur place dans l'ensemble des cultes à mystères. Également controversées, les particularitéss ethniques de leur origine et l'époque de leur apparition.

Dans cet ordre d'idées, il est à noter leur grande popularité à la période hellénistique et la place importante qu'ils occupent, surtout dans la Méditerranée orientale. Sur la base des données de la Thrace et de la côte thrace du Pont, qui présentent un intérêt tout particulier sous ce rapport, je voudrais analyser les monuments découverts et les informations qu'ils fournissent sur le caractère du culte et sur les raisons principales de sa propagation et de l'accroissement de son importance dans la région à cette époque.

Il convient d'aborder le problème de l'origine des mystères pour essayer de les situer dans le temps et d'évaluer leur place dans la religion de la Méditerranée orientale. En premier lieu se pose la question de l'ancrage ethnique de la population la plus ancienne de Samothrace. Certaines données écrites la lient à la tribu des Saïens. Les témoignages écrits les plus anciens sur cette tribu sont contradictoires. S'en tenant aux informations d'Archiloque (fr. 6), reprises plus tard par Strabon (XII, 3, 20), H. Danov ${ }^{2}$ définit les Saïens comme une tribu thrace qui habitait cette région de la côte égéenne et dont une partie continuait à habiter, à l'époque de la colonisation grecque, la région et certaines des îles, entre autres Samothrace. Il semble que

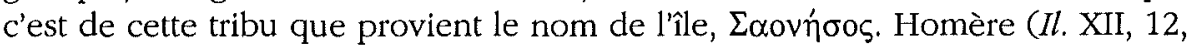
et Schol. ad 12-13) parle de "Samos thrace» et le scholiaste explique qu'Homère la distingue ainsi d'autres îles portant le même nom. Cela jette le

\footnotetext{
G.S. Cole, Theol Megaloi: The Cult of the Greatest Gods at Samothrace, Leiden,

2 H. Danov, Dreuna Trakia, Sofia, 1969, p. 156.
} 1984. 
doute sur la provenance du nom de l'île de oóor, en l'occurrence, de la tribu des Saïens, ainsi que sur l'appellation de la société mystérieuse que les Hellènes traduisent par le mot « sauvés » ${ }^{3}$.

Un autre témoignage sur cette période ancienne de l'histoire de l'île se trouve dans les Argonautiques d'Apollonios de Rhodes où les dieux de Samothrace passent pour des protecteurs de la navigation. C'est Orphée luimême qui conseille aux Argonautes d'implorer leur aide pour pouvoir continuer leur voyage (I, 916-918). Dans une scholie à ce texte, le nom de l'île se trouve expliqué par sa population la plus ancienne - un groupe de 40 Thraces. Une autre scholie identifie Samothrace à l'île d'Électre, la mère de Dardanos, le fondateur légendaire de Troie, ce qui relie l'île à la population de l'Asie Mineure. Au dire d'Aristote (fr. 579), l'éponyme de l'île aurait été Samon, fils d'Hermès et de la nymphe Rhéné. Si l'on fait une comparaison avec le témoignage d'Hérodote sur la vénération d'Hermès par les rois thraces, qui prétendent en descendre (V. 7), et sur Hermès, le père du fondateur légendaire de la ville thrace d'Abdère, on ne peut que constater la substitution des noms dans l'interprétation grecque du témoignage. C'est le même Hermès dont parle Hérodote dans un autre passage, directement lié à Samothrace, selon lequel les Athéniens ont été mis au courant du culte d'Hermès ithyphallique par les Hellènes, venus de Samothrace, qui connaissaient ce culte et ses mystères grâce aux Pélasges de l'île (II, 51) ${ }^{4}$. C'est un indice éloquent du caractère prégrec des mystères.

La question des Pélasges ne fait pas l'objet de la présente étude, car elle demeure en marge de l'analyse des mystères. Sans importance est aussi la question de savoir s'il s'agit d'une population thrace ou d'une population préthrace de l'île, d'autant plus que les informations sur ce point restent floues. Ce qui importe, en l'occurrence, ce sont l'origine non grecque des mystères et son lien catégorique avec une population sinon thrace, du moins prégrecque de la Méditerranée orientale, ainsi que ses contacts directs avec l'Asie Mineure.

Cette thèse se trouve étayée par les témoignages, d'ailleurs peu nombreux, sur le culte à mystères pratiqué dans l'île. C'est Hérodote qui est le premier à donner un nom aux dieux de Samothrace, les Cabires (II, 51), ce qui paraît étrange. Mais un peu plus loin (II, 52), il détermine catégoriquement les dieux des Pélasges comme anonymes, ce que les données confirment, surtout pour une époque ancienne. Dans les Argonautiques d'Apollonios de Rhodes, les dieux sont aussi anonymes (I, 916-918 et schol.). Or, le scholiaste essaie de les faire connaître au lecteur grec, quelque chose de typique pour l'époque hellénistique. Il donne beaucoup d'explications sur les Cabires et lie leur nom à l'Asie Mineure, notamment à une montagne homonyme en

3 W. BURKERT, Griechiscbe Religion der arcbaiscben und klassiscben Epocbe, Stuttgart, 1977, p. 285.

4 Zl. Gočmva, "Le culte d'Hermes en Thrace et le témoignage d'Hérodote V, 7", in Religion, Mytbologie, Iconographie, Rome, 1991, 159-166. 
Phrygie. Selon lui, ils sont au nombre de deux, un jeune Cabire, Dionysos, et un Cabire âgé, Zeus, ce dont témoignent les monuments votifs découverts en Phrygie, portant la représentation de deux divinités - une jeune divinité et une divinité âgée, mais sans inscription. Les noms concrets, donnés par le scholiaste à ces divinités, et leur identification avec la triade des mystères d'Éleusis sont en dehors du thème traité ${ }^{5}$. D. Detschew les voit comme des noms thraces ${ }^{6}$. L'étymologie qu'il propose ne saurait être expliquée par leur rôle dans les mystères. Plus importante est sa conclusion sur leur origine non grecque qui vient augmenter la crédibilité de notre thèse de l'origine non grecque des mystères. L'hypothèse de A. $\mathrm{Cook}^{7}$, avancée plus tard par L. Farnell ${ }^{8}$ et R. Pettazzoni ${ }^{9}$, que le nom de Cabires a été introduit par les commerçants phéniciens et traduit par les colons grecs comme Grands Dieux, est inacceptable. On ne peut pas admettre non plus les noms grecs, mentionnés par le scholiaste d'Apollonios de Rhodes, d'autant plus qu'il parle d'un culte thrace.

Les données les plus anciennes de l'exploration archéologique remontent à la période allant jusqu'au milieu du $\mathrm{IV}^{\mathrm{e}}$ siècle av. J.-C. ${ }^{10}$, mais il est possible d'obtenir d'autres datations par de nouvelles découvertes archéologiques. On commence à parler du sanctuaire des Grands Dieux de Samothrace et de son fonctionnement historique à l'époque hellénistique. C'est de cette époque qu'est datée aussi sa construction monumentale ${ }^{11}$. C'est tout à fait logique si l'on prend en considération son caractère et les particularités du culte pratiqué. Une autre explication en est le rôle important que jouent à l'époque hellénistique la culture et la religion méditerranéennes. La vénération de Grands Dieux, protecteurs de différentes villes, concorde avec la vénération de dieux anonymes dans cette région à une époque ancienne. Cela tient à cette particularité de la religion thrace qui n'obtient qu'à l'époque hellénistique une iconographie et une caractéristique plus concrètes de son dieu principal, le Cavalier thrace, qui demeure anonyme, mais se voit attribuer certaines épithètes fonctionnelles et, dans plusieurs cas, des appellations thraces locales qui le lient probablement à différentes régions. Ce n'est pas un hasard non plus si, dans quelques endroits, le Cavalier thrace porte aussi l'épithète de Théos Mégas ${ }^{12}$.

\footnotetext{
5 COLE, o.c. (n. 1), p. 2.

6 D. Detschew, Die thrakiscben Spracbreste, Wien, 1976, p. 18.

7 A.B. Cook, Zeus. A Study in Ancient Religion, New York, 1965, p. 313.

8 L.R. FARnelL, Greek Hero Cults and Ideas of Immortality, Oxford, 1921, p. 324.

9 R. Pettazzoni, "Les Mystères grecs et les religions à mystères de l'antiquité", Cabiers d'bistoire mondiale, Neuchâtel, 2, 1954-55, p. 310.

10 Cole, o.c. (n. 1), p. 12 sq.

11. Ibid.

12 Zl. GočkvA, "Les épithètes du Cavalier Thrace", Linguistique Balkanique 35 (Sofia, 1992), $3-4$, p. 155.
} 
Le sanctuaire de Samothrace connaît son premier essor au rve siècle av. J.C. Il devient populaire grâce à l'attention que lui prêtent les Macédoniens ${ }^{13}$. Tout d'abord, il faut mentionner Philippe de Macédoine et Olympias, et le récit de Plutarque (Alex., 2, 2) illustrant leur connaissance du sanctuaire. Philippe et, par la suite, Alexandre le Grand lui donnent de la terre sur la côte opposée. Selon Philostrate (Vie d'Apoll. Tyane II, 43), Alexandre fait même construire un autel, dédié aux dieux de Samothrace, sur la frontière avec l'Inde. On a découvert beaucoup de monuments épigraphiques dans le sanctuaire de cette époque. Dans une des inscriptions est mentionné Philippe III Aridaios $^{14}$. La popularité du sanctuaire à cette époque peut être expliquée, en général, par le désir d'opposer les mystères de Samothrace aux mystères d'Éleusis. La raison en est la remise à honneur des cultes prégrecs anciens dans la région, un trait caractéristique de la politique culturelle de l'hellénisme qui conditionne son dynamisme. Ce n'est pas un hasard si ces cultes rencontrent l'intérêt des dynastes macédoniens du fait d'être plus proches de leurs idées religieuses. La construction monumentale du sanctuaire est à associer aux Diadoques ${ }^{15}$. À Lysimaque sont consacrées deux inscriptions du sanctuaire qui nous informent au sujet d'un autel, édifié en son honneur, et d'un sacrifice annuel. Son épouse Arsinoé aurait été initiée aux mystères et c'est à elle qu'on lie l'édifice circulaire insolite au centre du sanctuaire. Les grands propylées sont construits par son frère et son troisième époux ${ }^{16}$. Parmi les décrets honorifiques, on distingue ceux de deux chefs militaires de Ptolémée III Évergète, Hippomédon et Érinyce. Au centre du sanctuaire, les Macédoniens ont érigé une statue de Philippe V. Tout cela témoigne du fait que le sanctuaire est un centre cultuel important de la religion hellénistique dans cette région, lié à un culte ancien, bien connu de la population hellénistique et des familles royales.

Aussi est-il logique de voir apparaître le culte des dieux de Samothrace dans le milieu thrace, proche de ces idées religieuses, qu'est la capitale de Seuthès III, Seuthopolis ${ }^{17}$. A la fin d'un décret, signé par l'épouse de Seuthès III, Bérénice, et ses fils, et par le roi de Cabylé, Spartocos, on peut lire qu'il faut déposer l'un des exemplaires de l'inscription dans-le sanctuaire des dieux de Samothrace à Seuthopolis ${ }^{18}$. C'est le monument le plus ancien qui témoigne de l'existence d'un sanctuaire de ces dieux en dehors de

\footnotetext{
13 COLE, o.c. (n. 1), p. 18 sq.

14 Ibid.

15 Ibid., p. 21

16 G. Bengtson, Prawiteli epobi elinizma, Moskwa, 1982, p. 139-160; Cole, o.c. (n. 1), p. 22-23.

17 D.P. Dimitrov, "Das Entstehen der thrakischen Stadt und die Eigenart ihrer städtebaulicher Gestaltung und Architektur", Antiquity 35 (1961), n 138.

18 D. Dimitrov, "Kam vaprosa za religijata na trakite ot ranoelinisticeskata epoha" [en bulgare], Istoriceski pregled 23 (1957), 2, p. 65-81; G. Minailov, "La Thrace et la Macédoine jusqu'à l'invasion des Celtes", Arbaia Makedonia I, Thessaloniki, 1970, p. 84.
} 
Samothrace. L'inscription a été découverte in situ dans la grande salle du palais de Seuthès III. La propagation du culte de ces dieux à Seuthopolis a suscité des interprétations diverses. K.-L. Elvers affirme qu'il s'agit d'un culte étranger, importé dans la ville, d'un culte adopté comme une mode par la famille de Seuthès, qui aurait été pratiqué plutôt par la population grecque de Seuthopolis ${ }^{19}$. Cette hypothèse semble être tout à fait aventureuse. Tout d'abord, il s'agit d'un monument officiel qu'il faut rechercher dans les archives lapidaires de la ville, lesquelles suggèrent l'existence d'un temple principal et d'un culte pratiqué par sa population. On n'est pas fondé à parler de masses d'étrangers dans la ville et à les associer à sa religion officielle. Il n'y a pas de données sur la pratique du culte des dieux de Samothrace comme un culte grec, en général, dans cette région de la Méditerranée, ce dont témoigne son histoire à une époque plus ancienne. Selon M. Tatscheva, il s'agit de dieux « étrangers depuis longtemps » à la religion thrace, mais qui sont adoptés comme une mode dans le monde hellénistique ${ }^{20}$. Cette hypothèse ne cadre en aucune façon avec la ferveur que montrent les Thraces dans la pratique de leur religion. Bien au contraire, leur culte est adopté et pratiqué en raison de ses affinités avec les idées religieuses de la Méditerranée orientale d'une époque ancienne, dont fait partie le culte des Grands Dieux de Samothrace. On ne saurait parler nullement d'hellénisation de la culture thrace, car il s'agit d'une époque hellénistique où les Thraces ont leur propre place. On ne saurait parler non plus de vénération des dieux de Samothrace dans la citadelle seulement par le roi, indépendamment de la population. De l'union de toute la population et du roi témoignent aussi les eschara, découverts en plusieurs endroits dans la ville ${ }^{21}$.

Une autre aire de diffusion du culte des Grands Dieux de Samothrace est la partie nord-ouest de la côte pontique. Il y eut des tentatives d'associer le culte des différents Grands Dieux, protecteurs des villes pontiques, aux Grands Dieux de Samothrace ${ }^{22}$ : à Odessos ou à Histria, dans une inscription qui nous informe au sujet de la vénération d'un Grand Dieu anonyme avec un temple monumental. Or, c'est une question délicate. À Odessos, une telle association est douteuse. C'est sur la base d'un des décrets, découverts dans

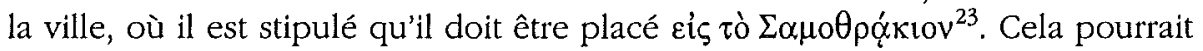
être un témoignage de l'existence d'un temple des Grands Dieux de Samothrace des III $^{\mathrm{e}}-\mathrm{II}^{\mathrm{e}}$ siècles. G: Mihailov considère qu'il s'agit peut-être d'un temple commun des Grands Dieux de Samothrace et du Théos Mégas

19 K.-L. Elvers, "Der Eid der Berenike und ihrer Söhne: eine Edition von IGBulg III, 2, 1731", Chiron 24 (1994), p. 264.

20 M. Tatscheva, Seutbes III, Séutopois und Kabyle (341-252 v. Cbr.) nacb den epigrapbiscben und numismatiscben Angaben, Sofia, 2000, p. 40-41.

21 Dimitrov, l.c. (n. 18).

22 A. SAlač, "Le grand dieu d'Odessos - Varna et les mystères de Samothrace", $B C H$ 52 (1928), p. $397 s q$.

23 G. MuHalLov, Inscriptiones Graecae in Bulgaria (IGBulg) I, Sofia, 1970, $\mathrm{n}^{\circ} 42$. 
d'Odessos, en ajoutant les Cabires qui sont attestés aussi par une inscription d'Odessos. On n'est nullement fondé à lier ces trois cultes différents. M. Mirčev va même jusqu'à localiser ce temple à Odessos sur la base des matériaux votifs, découverts in situ, consacrés aux Cabires ${ }^{24}$. Le lien d'Odessos avec le sanctuaire des Grands Dieux de Samothrace se trouve aussi confirmé par un décret, découvert à Samothrace, qui nous informe de la participation aux mystères de Samothrace d'habitants d'Odessos ${ }^{25}$. C'est à nouveau une preuve de la pratique du culte des dieux de Samothrace par les habitants d'Odessos et de l'existence de leur temple. Selon L. Robert ${ }^{26}$, le prêtre d'Odessos, mentionné dans le décret (dont le nom manque), est un prêtre-éponyme qui aurait été aussi un prêtre du Grand Dieu d'Odessos. Or, le texte de l'inscription ne permet pas d'aboutir à une telle conclusion. Il n'y a pas de données sur son lien éventuel avec les mystères de Samothrace et sa mention ne pourrait servir, en l'occurrence, que de formule de datation.

Le culte des dieux de Samothrace est attesté plus catégoriquement à Histria. Dans un fragment d'une inscription, datée du $\mathrm{II}^{\mathrm{e}}$ siècle avant J.-C., il est dit qu'elle doit être placée $\dot{\varepsilon} v \tau \hat{\varphi} \Sigma \alpha \mu \mathrm{o} \theta \rho \alpha \kappa i \omega^{27}$. Dans un autre décret honorifique, on peut lire qu'il faut le déposer $\pi \alpha \rho \dot{\alpha} \tau$ ìv $\beta \omega \mu$ òv $\tau \hat{\omega} v \theta \varepsilon \hat{\omega} v \tau \hat{\omega} v \dot{\varepsilon} v$ $\Sigma \alpha \mu \mathrm{o} \theta \rho \alpha_{\alpha}^{\prime} \kappa ı \eta \imath^{28}$. Selon D. Pippidi, il s'agit de la même chose. Une information intéressante est fournie par un autre décret d'Histria, dans laquelle on peut

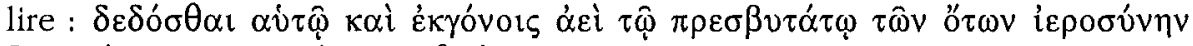

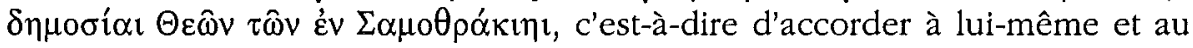
plus âgé de ses descendants le sacerdoce des Grands Dieux de Samothrace, déterminé aussi comme un culte officiel ${ }^{29}$. Tout cela conduit $\mathrm{D}$. Pippidi à supposer qu'il s'agit d'une personne ayant de grands mérites à l'égard du culte et, probablement, du fondateur de ce culte à Histria ou de l'un de ses premiers fidèles. Il réfute lui-même sa première hypothèse en admettant que la pénétration du culte des dieux de Samothace à Histria aurait coïncidé avec la période de la propagation de ce culte dans cette partie de la Péninsule balkanique à l'époque hellénistique. Or, il confond les dieux de Samothrace et les Cabires en se référant à D. Dimitrov ${ }^{30}$ et à l'inscription de Seuthopolis, bien que les Cabires ne soient mentionnés ni dans l'inscription ni dans la publication de D. Dimitrov.

D'un temple des Grands Dieux de Samothrace témoigne aussi un décret, récemment découvert à Dionysopolis, par lequel est honoré un habitant de

24 M. MrRčev, "Za antiènite hramove v Odesos" [en bulgarie], Izw. na Narodnija Muz. v. Varna 3 (1967), p. 21-39.

25 Mihailov, o.c. (n. 23), p. 92-93.

26 L. ROBERT, "Les inscriptions grecques de Bulgarie", RPb 33 (1959), p. 191-193.

27 D. PIppIDI, Inscriptile din Scythia Minor grecešti ši latine V, Bucaresti, $1983, \mathrm{n}^{\circ} 58$.

28 Ibid., $\mathrm{n}^{\circ} 11$.

29 Ibid., $\mathrm{n}^{\circ} 19$.

30 Dimitrov, l.c. (n. 18), p. 65. 
Messambria et il est dit de déposer l'inscription dans le sanctuaire des Grands Dieux de Samothrace. Sur trois fragments de stèles de Callatis, on peut lire aussi qu'elles doivent être placées dans le sanctuaire des Grands Dieux de Samothrace.

Les matériaux présentés permettent d'aboutir à la conclusion suivante. La pénétration du culte des Grands Dieux de Samothrace en Thrace a suscité différentes hypothèses. Selon D. Dimitrov, qui a publié l'inscription de Seuthopolis, on pourrait parler d'un lien direct avec leur sanctuaire à Samothrace. G. Mihailov affirme que leur culte aurait été amené à Seuthopolis par l'intermédiaire des colonies pontiques grecques ${ }^{31}$. Cette hypothèse paraît insoutenable du fait que l'inscription de Seuthopolis est le témoignage le plus ancien de la pénétration de leur culte. Si l'on admet que ce culte est adopté à Seuthopolis du fait qu'il est proche des Thraces, c'est d'ailleurs l'hypothèse la plus plausible, il n'y a pas de doute que sous l'influence thrace, à la fin de l'époque hellénistique, s'est créé comme une communauté politico-religieuse qui se propose de garantir une certaine indépendance des idées religieuses traditionnelles. Odessos, qui est aussi la première capitale du koinon pontique $^{32}$, est une ville qui se distingue à cette époque par la grande influence locale sur le développement de sa vie religieuse, attestée par la pénétration du culte du Cavalier thrace et par le syncrétisme du Grand Dieu d'Odessos et du dieu thrace Darzalas. Tout cela vient réfuter la possibilité d'une introduction du culte des dieux de Samothrace en Thrace et sur la côte pontique en tant que clause du traité de paix signé avec Lysimaque, comme l'affirme K. Rabadziev. Une telle ingérence brutale dans la vie religieuse de la population locale n'est pas typique de l'époque hellénistique. On peut parler de processus plus complexes. Les noms grecs de certains prêtres du dieu à l'époque hellénistique, surtout à l'époque romaine, ne sont pas obligatoirement d'origine hellénique. Il n'y a pas de doute que la population thrace s'associe au culte pratiqué dans un sanctuaire qui maintient les traditions religieuses prégrecques de la Méditerranée auxquelles les idées religieuses thraces sont étroitement liées.

Zlatozara GočEvA

Institut de thracologie

Académie Bulgare des sciences

13 rue Moskovska

BG - 1000 SofIA

31 Mihallov, o.c. (n. 23), p. 92 sq.

32 Zl. Gočeva, "Organization of the religious and administrative live of the western Pontic Koinon", Thracia 12 (1998), p. 141-146. 